Journal of Animal and Veterinary Advances 11 (2): 191-199, 2012

ISSN: $1680-5593$

(C) Medwell Journals, 2012

\title{
Quality Control and Discrimination of Different Processed Products of Angelica Based on High-Performance Liquid Chromatographic Fingerprints Combined Chemometrics Methods
}

\author{
${ }^{1} \mathrm{Hua}$ Yong-Li, ${ }^{1,2}$ Guo Yan-Sheng, ${ }^{2} \mathrm{He}$ Sheng-Hu, ${ }^{1} \mathrm{Fan}$ Jiang-Feng and ${ }^{1} \mathrm{Wei}$ Yan-Ming \\ ${ }^{1}$ School of Veterinary Medicine, Gansu Agriculture University, 730070 Lanzhou, China \\ ${ }^{2}$ School of Agriculture, Ningxia University, 750021 Yinchuan, China
}

\begin{abstract}
A chemical fingerprint method was developed for reorganizing and validating different processed products of Angelica using HPLC. About 11 common peaks of this HPLC fingerprints were found by assigning Ferulic acid peak as the reference peak. The correlation coefficient of entire chromatographic patterns among samples were calculated and the simulative mean chromatogram was calculated and generated using the Computer Aided Similarity Evaluation System, the observation indicated that the characteristic peaks of this HPLC fingerprints existed significant difference between different processed products. Data were evaluated statistically using Principal Component Analysis (PCA), Hierarchical Cluster Analysis (HCA) and Discriminant Analysis (DA) in order to classify the samples and to identify key categorizing parameters. The statistical results showed that the projected plots of each processed products were localized in confined cluster in the 3D-projection plots of Principal Component Analysis ( $\mathrm{PCA}$ ) and that 55 representative samples were separated into 5 groups using HCA at a rescaled distance of 5 and the same processed product samples were clustered in the same cluster. Furthermore, Fish's discriminant functions were generated using 6 selected predictor variables, the tested samples of different processed products were classified with $100 \%$ accuracy and DA plots for the 5 groups were well-resolved. To the knowledge, this is the first demonstration of the feasibility and advantages of employing chromatographic fingerprinting combined with PCA, HCA and DA for the accurate identification and validation of different processed products of Angelica. The methodology can also be used to determine relationships between chemical composition and therapeutic effects and assess quality of different processed products of Angelica.
\end{abstract}

Key words: Angelica, different processed products, fingerprints, Chemometrics Methods, data, China

\section{INTRODUCTION}

Chinese Angelica (Radix Angelicae sinensis), the root of Angelica sinensis (Oliv.) Diels is mainly cultivated in Minxian, Gansu province, China. It has been used for thousands of years in traditional Chinese which was firstly cited in Shenlong Bencao Jing, a classical masterpiece of Traditional Chinese Medicines (TCM). It is predominantly renowned for its use in the treatment of a wide variety of gynecological conditions that are generally not easily treated with conventional therapies which has been called female ginseng. Besides that it has been widely applied to treat anemia, constipation, cardiovascular disease and hepatic fibrosis. What is more, its medicinal value has been demonstrated by numerous clinical trials, pre-clinical studies and traditional or modern experiments. Chinese herbal medicine processing is a traditional pharmaceutical technology for further processing Chinese herbal medicine according to the theory of TCM. Processing decoction pieces are used as not only one of the herbal ingredients in prescriptions of TCM but also the raw medicine of TCM preparation. Hence, its quality are directly related therapeutic effect so that all physicians in the past dynasties has been always highly regarded Chinese herbal medicine processing. At present, the processed products of Angelica mainly includes Charred Angelica, Parching Angelica with spice oil, Parching Angelica with wine and Parching Angelica with soil which have been widely in prescriptions of TCM. The related researchers found that different processed product of Angelica has different efficacy. This reason may be explained from the chemical change of different processing product of Angelica. Therefore, developing a simple, fast and sensitive methodology to analyze chemical change has important theoretical significance and wide application foreground for explaining the principal of Angelica processing and making quality standard of Angelica processed products.

Corresponding Author: Wei Yan-Ming, School of Veterinary Medicine, Gansu Agriculture University, 730070 Lanzhou, China 
Now-a-days, chromatographic fingerprints are being widely used in quality control of Danggui, there is some publications discussed about fingerprint of volatile components by GC-MS and non-volatile components by HPLC-DAD or HPLC-MS. Furthermore, the Computer Aided Similarity Evaluation System developed by the Research Center of Modernization of Traditional Chinese Medicines (Central South University, Changsha, China) was applied as a very useful for quantitatively studies of the similarity of chromatographic patterns and generation of the mean chromatogram use as standard chromatogram of samples. With the help of chemometric methods, such as correlation coefficient and Principal Component Analysis (PCA), the developed HPLC fingerprints can be used to differentiate Chinese Angelica, Japanese Angelica, Szechwan Lovage Rhizome and Cnidium Rhizome.

The chemical components in Chinese Angelica and Japanese Angelicae root were quite different even though they belong to same genus, genus Angelica and both of them are official medicinal materials used in China and Japan, respectively.

Chromatographic fingerprints are very sensitive to distinguish these similar herbs. Wang et al. (2006) established a criterion for distinguishing angelica from the three areas with chromatographic fingerprint combined with discriminant analysis, the accuracy of the Discriminant Model is as high as $90 \%$ which is much better than guessing or traditional methods. Recently in order to investigate the chemical components of whole roots and prepared slices of Angelica, HPLC combined with Hierarchical Cluster Analysis (HCA) was applied to provide amore detailed study by Wu et al. (2008). All these researches indicated that chemometric methods are absolutely necessary for the application of chromatographic fingerprint. However, although chromatographic fingerprint analysis provides a highly rational approach to the quality assessment of Angelica, to the knowledge it has not previously been applied to the identification and classification of Angelica processed products. Therefore, the aim of the present study was to develop simple, fast and sensitive fingerprinting system with Chemometric Methods to differentiate Angelica different processed products and make their quality stadards.

\section{MATERIALS AND METHODS}

Original medicinal material and processed products: The indentity, sampling part and sample source of 11 tested samples are shown in Table 1. These herbal samples were authenticated by Dr. Yan-Ming Wei (School of Veterinary Medicine, Gansu Agriculture University, Lanzhou, China). Each of representative samples was cut into smaller pieces and further equally divided into 5 groups. About 1 group was used as unprocessed Angelica sample the other four groups were respectively processed as Charred Angelica, Parching Angelica with spice oil, Parching Angelica with wine and Parching Angelica with soil samples according to Pharmacopoeia Commission of the People's Republic of China and Gan Su Processing standard of traditional Chinese medicine (Table 1). Voucher specimens are stored the Herbarium Center of this institution. All samples were ground into powder and passed through a 40 mesh sieve and the ground powders were stored at about 4 .

Solvents and chemicals: Analytical grade methanol () and formic acid () were used for sample preparation. HPLC grade acetonitrile (), deionized water obtained from a Milli-Q water system (Millipore, Bedford, MA, USA) and analytical grade glacial acetic acid () were used for preparation of mobile phase.

Reference compounds preparation: Ferulic acid was purchased from the Institute for the Control of Pharmaceutical and Biological Products of China (Beijing, China). About $4.8 \mathrm{mg}$, ferulic acid was dissolved and diluted to $100 \mathrm{~mL}$ with methanol.

Table 1: Origins of Angelic a sinensis (Oliv). Diels from Gansu and sample code of processed products

\begin{tabular}{|c|c|c|c|c|c|c|c|}
\hline \multirow[b]{2}{*}{$\begin{array}{l}\text { Sample } \\
\text { code }\end{array}$} & \multirow[b]{2}{*}{ Sources } & \multirow[b]{2}{*}{ Sampling part } & \multirow[b]{2}{*}{ Date of collection } & \multicolumn{4}{|c|}{ Code of processed products } \\
\hline & & & & Charring & $\begin{array}{c}\text { Parching with } \\
\text { spice oil }\end{array}$ & $\begin{array}{l}\text { Parching } \\
\text { with wine }\end{array}$ & $\begin{array}{l}\text { Parching } \\
\text { with soil }\end{array}$ \\
\hline$\overline{\mathrm{S} 1}$ & Jinzhongzhen, Zhangxian & Whole root & September, 2008 & $\mathrm{~T} 1$ & Y1 & $\mathrm{J} 1$ & TU1 \\
\hline S2 & Zhongzhaizhen, Minxian & Whole root & September, 2008 & $\mathrm{~T} 2$ & $\mathrm{Y} 2$ & $\mathrm{~J} 2$ & TU2 \\
\hline $\mathrm{S} 3$ & Xizhaizhen, Minxian & Whole root & September, 2008 & $\mathrm{~T} 2$ & Y3 & $\mathrm{J} 3$ & TU3 \\
\hline S4 & Mazichuanxiang, Minxian & Whole root & September, 2008 & $\mathrm{~T} 4$ & $\mathrm{Y} 4$ & $\mathrm{~J} 4$ & TU4 \\
\hline S5 & Tianjiahezhen, Weiyuan & Whole root & September, 2008 & T5 & Y5 & J5 & TU5 \\
\hline S6 & Wuzhuzhen, Weiyuan & Whole root & September, 2008 & T6 & Y6 & J6 & TU6 \\
\hline S7 & Huichuanzhen, Minxian & Whole root & September, 2008 & $\mathrm{~T} 7$ & Y7 & $\mathrm{J} 7$ & TU7 \\
\hline S8 & Dacaotanxiang, Zhangxian & Whole root & September, 2008 & $\mathrm{~T} 8$ & Y8 & J8 & TU8 \\
\hline S9 & Shichuanxiang, Zhangxian & Whole root & September, 2008 & T9 & Y9 & J9 & TU9 \\
\hline $\mathrm{S} 10$ & Shilixiang, Minxian & Whole root & September, 2008 & $\mathrm{~T} 10$ & $\mathrm{Y} 10$ & $\mathrm{~J} 10$ & TU10 \\
\hline $\mathrm{S} 11$ & Minxian market of Chinese herb & Whole root & September, 2008 & $\mathrm{~T} 11$ & $\mathrm{Y} 11$ & $\mathrm{~J} 11$ & TU11 \\
\hline
\end{tabular}


Sample preparation: An accurately weighed sample powder of $0.5 \mathrm{~g}$ was transferred to $50 \mathrm{~mL}$ erlenmeyer flask and $25 \mathrm{~mL}$ methanol-formic (95:5) was added. The weight of vial was record and the erlenmeyer flask was sealed and sonicated for $60 \mathrm{~min}$. The original solvent weight was restored. The extract was filtered through a $0.45 \mu \mathrm{m}$ membrane filter. An aliquot of $10 \mathrm{~mL}$ solution was injected for HPLC analysis.

HPLC conditions: Samples chromatograms were acquired using an Aglient/HP 1100 series HPLC-UV system consisting of a vacuum degasser, binary pump, autosampler, thermosatated column compartment and binary wavelength detector (Aglient, Palo Alto, CA, USA) and fitted with a and fitted with a ODS- $\mathrm{C}_{18}$ reversed-phase column $(5 \mu \mathrm{m}, 4.6 \mathrm{~mm} \times 250 \mathrm{~mm})$. The mobile phase consisted of $1 \%$ acetic acid in water (A) and acetonitrile (B) using a gradient program of $20 \sim 30 \%$ (B) in $0 \sim 10 \mathrm{~min}$, $30 \sim 49 \%$ (B) in 10 20 min, 49\% (B) in 20 40 min; 49 100\% (B) in $40 \sim 50 \mathrm{~min} ; 100 \sim 20 \%$ (B) in $50 \sim 60 \mathrm{~min}$. The

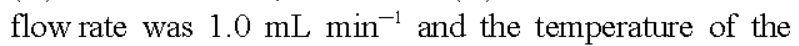
column was maintained at $25^{\circ} \mathrm{C}$. The detection wavelength was set at $280 \mathrm{~nm}$ and the total recording time was $60 \mathrm{~min}$.

Data analysis of chromatogram: Data analysis was performed using Computer Aided Similarity Evaluation (CASE) software as recommended by the Chinese Pharmacopoeia Committee. The software is used for evaluating similarities between different chromatograms and generating simulative mean chromatogram. The RRT and RPA of each characteristic peak to reference peak were also calculated in the chromatograms. Three principal components obtained by PCA were used to evaluate the similarities and differences among the test samples. HCA and DA were performed using SPSS software (SPSS for Windows 13.0, SPSS Inc., USA).

\section{RESULTS AND DISCUSSION}

Selction of chromatographic conditions: Ferulic acid are commonly found in Angelica and its different processed products, it was assigned as marker in State Pharmacopoeia Commission of the People's Republic of China (2005) for assessing the quality of Angelica owing to its bioactivity and present in relatively stabile peak area. Therefore, ferulic acid was chosen as characteristic compounds for detection.

Regarding the choice of solvent for optimal extraction, organic solvents including hexane, aqueous methanol and ethylacetate-methanol (70:30) were used for developing angelica fingerprints in previous studies. Methanol-formic acid (95:5) was the preferred choice of extraction solvent in the present study as a variety of compounds with different polarity can be extracted effectively and were relatively stable in this organic solvent. Besides, the interference from sugars in the raw herbs could also be minimized by extraction using methanol-formic acid (95:5). Therefore, extraction using methanol-formic acid (95:5) was chosen in this study.

Selection of detection wavelength was one of the key factors contributing to a reliable and reproducible HPLC fingerprint of Angelica and its different products. The wavelengths of $210,320,270$ or $284 \mathrm{~nm}$ were used for detection in published Angelica HPLC fingerprint and multi-components analyses. In this study, good peak separation was achieved at the wavelength of $250 \mathrm{~nm}$. Hence, characteristic chromatographic patterns were obtained to distinguishing angelica and its different processed products.

Method reproducibility and repeatability were evaluated by the analysis of six injection of sample solution and six replicates of solid samples, respectively. Precision of retention times and peaks areas of compounds 1-11 for replicated injection were found in the range of $0.03-0.14$ and $0.54-2.06 \%$ of $\operatorname{RSD}(n=5)$, respectively. The RSD of peak area of compound 1-11 in sample replicates were estimated to be $0.75-1.82 \%(n=6)$. All results indicated that the conditions for the fingerprint analysis were satisfactory.

HPLC fingerprints of angelica and its processed products: The HPLC fingerprints were obtained by analyzing 55 Angelica and its different processed products samples. About 11 common peaks were found in these HPLC fingerprints according to the RRT (Fig. 1 and 2). Peaks 1 were unequivocally identified as ferulic acid by spiking authentic compound. Owing to the unavailability of authentic compounds, peaks 2-11 could only be tentatively assigned as Senkyunolide I (The Compile Commission of Zhonghua Bencao of the People's Republic of China, 1999), Senkyunolide H, Unkown compounds (Chen, 2008), Coniferyl ferulate (Zhang et al., 2007), Senkyunolide A (Wu et al., 2008) Butylphthalide (State Pharmacopoeia Commission of the People's Republic of China, 2005), E-ligustilide (Gansu Province Health Department, 1999), Z-ligustilide (Lu et al., 2005), Z-butylidenephthalide (Yi et al., 2007a), Levistolide A (Yi et al., 2007b) by comparing UV spectra of those compounds and those reported in literatures. For unambiguous identification of these compounds, further studies are required by using authentic compounds.

\footnotetext{
Distinguishing of Unprocessed Angelica and Charred Angelica: The correlation coefficients of each chromatogram within groups Unprocessed Angelica and Charred Angelica to corresponding software-generated group simulative mean chromatograms and the correlation
} 


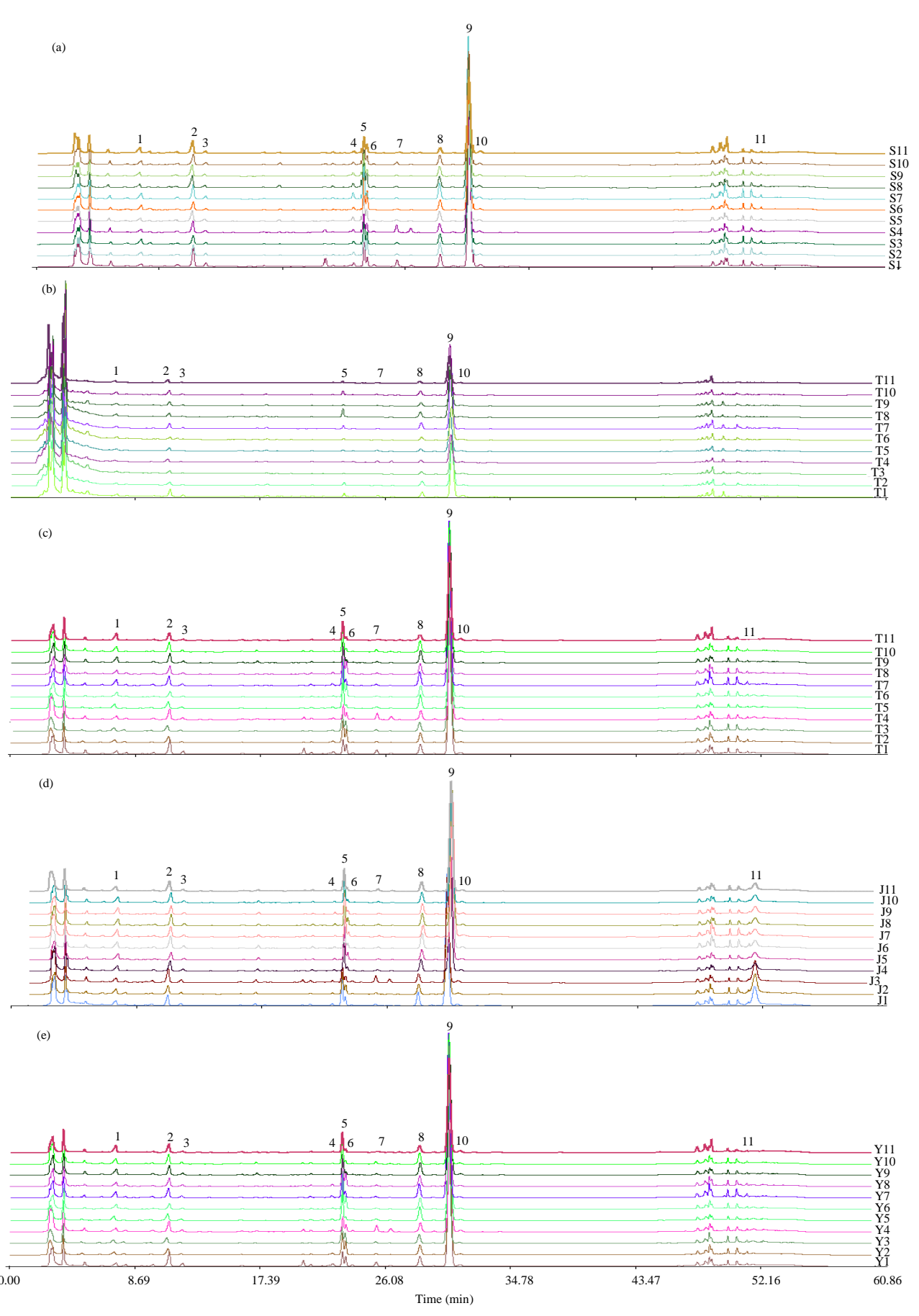

Fig. 1: HPLC fingerprint graphics of; a) Unprocessed Angelica; b) Charred Angelica; c) Parching Angelica with spice oil; d ) Parching Angelica with wine and e) Parching Angelica with soil. Analytical column: ODS- $\mathrm{C}_{18}, 4.6 \mathrm{~mm} \times 25$ $\mathrm{cm}, 5 \mu \mathrm{m}$; injected sample volume. About $10 \mu \mathrm{L}$; mobile phase: $1.0 \%$ acetic acid in water (A) and acetonitrile (B) using a gradient program of $20 \sim 30 \%$ (B) in $0 \sim 10 \mathrm{~min}, 30 \sim 49 \%$ (B) in $10 \sim 20 \mathrm{~min}, 49 \%$ (B) in $20 \sim 40 \mathrm{~min}$; 49 100\% (B) in $40 \sim 50 \mathrm{~min}$; $100 \sim 20 \%$ (B) in 50 60 min; flow rate: $1 \mathrm{~mL} \mathrm{~min}^{-1}$; temperature: $25^{\circ} \mathrm{C}$; UV detection: $280 \mathrm{~nm}$

coefficients between these simulative mean patterns within groups Unprocessed Angelica and chromatograms are shown in Table 2. Chromatographic Charred Angelica were generally consistent within each 

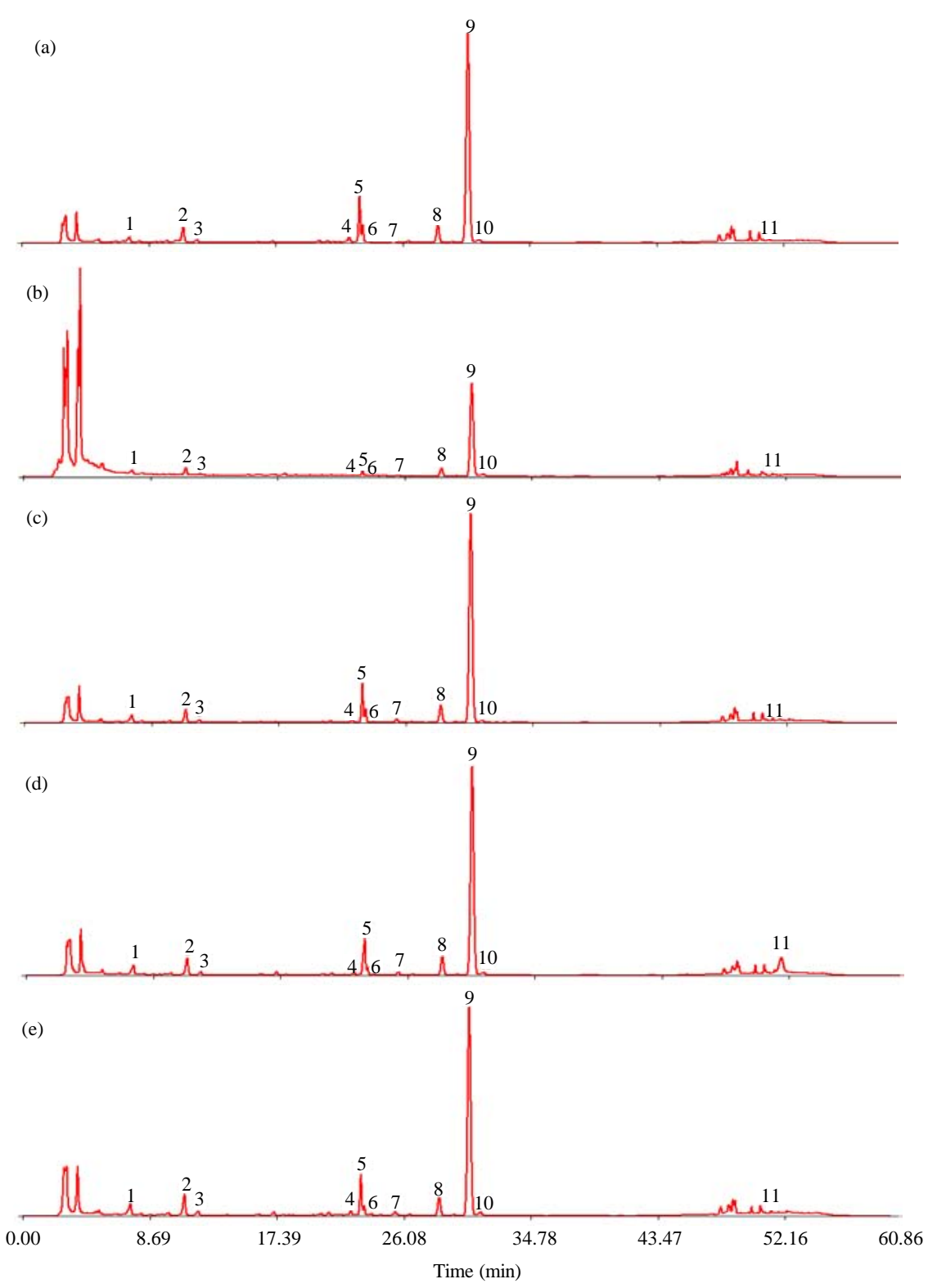

Fig. 2: Standard chromatographic project (Scp) of; a) Unprocessed Angelica; b) Charred Angelica; c) Parching Angelica with spice oil; d) Parching Angelica with wine and e) Parching Angelica with soil. Ferulic acid (1); Senkyunolide I (2); Senkyunolide H (3); Unkown compounds (4); Coniferyl ferulate (5); Senkyunolide A (6); Butylphthalide (7); E-ligustilide (8); Z-ligustilide (9); Z-butylidenephthalide (10) and Levistolide A (11)

Table 2: Similarity comparison of the chromatographic pattern between different processed products of Angelica sinensis (Oliv.) Diels

\begin{tabular}{llllll}
\hline Samples & $\mathrm{S}$ & $\mathrm{T}$ & $\mathrm{Y}$ & $\mathrm{J}$ & $\mathrm{TU}$ \\
$\mathrm{S}$ & $0.991 \pm 0.009^{\mathrm{a}}(\mathrm{n}=11)$ & $0.549^{\mathrm{b}}$ & $0.994^{\mathrm{b}}$ & $0.979^{\mathrm{b}}$ & $0.986^{\mathrm{b}}$ \\
$\mathrm{T}$ & - & $0.968 \pm 0.014^{\mathrm{a}}(\mathrm{n}=11)$ & $0.520^{\mathrm{b}}$ & $0.522^{\mathrm{b}}$ & $0.561^{\mathrm{b}}$ \\
$\mathrm{Y}$ & - & - & $0.995 \pm 0.003^{\mathrm{a}}(\mathrm{n}=11)$ & $0.99^{\mathrm{b}}$ & $0.993^{\mathrm{b}}$ \\
$\mathrm{J}$ & - & - & - & $0.970 \pm 0.016^{\mathrm{a}}(\mathrm{n}=11)$ & $0.984^{\mathrm{b}}$ \\
$\mathrm{TU}$ & - & - & - & - & $0.964 \pm 0.029^{\mathrm{a}}(\mathrm{n}=11)$ \\
\hline
\end{tabular}

S: Unprocessed Angelica; T: Charred Angelica; Y: Parching Angelica with spice oil; J: Parching Angelica with wine; TU: Parching Angelica with soil. ${ }^{a}$ The correlation coefficient of each chromatogram to themselves simulative mean chromatogram, mean $\pm \mathrm{SD}$. The correlation coefficient between mean chromatograms 
Table 3: The Relative Peak Aear (RPA) of characteristic peaks in different processed products

\begin{tabular}{llllllll} 
& \multicolumn{3}{c}{ The Relative Peak Aear (RPA) } \\
Peak no. & Compounds & $\begin{array}{c}\text { Rention time } \\
\left(\mathrm{t}_{\mathrm{R}} \mathrm{min}^{-1}\right)\end{array}$ & Unprocessed & Charring & $\begin{array}{l}\text { Parching with } \\
\text { spice oil }\end{array}$ & $\begin{array}{l}\text { Parching } \\
\text { with wine }\end{array}$ & Parching with soil \\
\hline 1 & Ferulic acid & 7.43 & 1.00 & 1.00 & 1.00 & 1.00 & 1.00 \\
2 & Senkyunolide I & 11.12 & $1.95 \pm 0.321$ & $0.88 \pm 0.086$ & $1.41 \pm 0.244$ & $1.54 \pm 0.265$ & $1.55 \pm 0.172$ \\
3 & Senkyunolide H & 12.04 & $0.45 \pm 0.084$ & $0.60 \pm 0.070$ & $0.30 \pm 0.050$ & $0.37 \pm 0.097$ & $0.45 \pm 0.059$ \\
4 & Unkown compounds & 22.46 & $0.62 \pm 0.161$ & $0.15 \pm 0.054$ & $0.24 \pm 0.060$ & $0.21 \pm 0.044$ & $0.31 \pm 0.048$ \\
5 & Coniferyl ferulate & 23.15 & $3.15 \pm 0.962$ & $0.42 \pm 0.099$ & $2.29 \pm 0.625$ & $2.59 \pm 0.304$ & $2.00 \pm 0.604$ \\
6 & Senkyunolide A & 23.83 & $0.00 \pm 0.000$ & $0.20 \pm 0.060$ & $0.12 \pm 0.027$ & $0.14 \pm 0.062$ & $0.17 \pm 0.062$ \\
7 & Butylphthalide & 25.48 & $0.38 \pm 0.089$ & $0.22 \pm 0.088$ & $0.29 \pm 0.099$ & $0.22 \pm 0.057$ & $0.29 \pm 0.053$ \\
8 & E-ligustilide & 28.51 & $2.17 \pm 0.423$ & $0.57 \pm 0.158$ & $2.06 \pm 0.307$ & $1.53 \pm 0.190$ & $1.47 \pm 0.233$ \\
9 & Z-ligustilide & 30.56 & $26.10 \pm 3.012$ & $8.00 \pm 1.341$ & $28.39 \pm 4.481$ & $19.42 \pm 3.011$ & $17.78 \pm 3.271$ \\
10 & Z-butylidenephthalide & 31.30 & $0.50 \pm 0.336$ & $0.24 \pm 0.072$ & $0.31 \pm 0.058$ & $0.31 \pm 0.064$ & $0.37 \pm 0.084$ \\
11 & Levistolide A & 51.66 & $0.00 \pm 0.000$ & $0.15 \pm 0.019$ & $0.86 \pm 0.391$ & $2.21 \pm 0.330$ & $0.55 \pm 0.166$ \\
\hline
\end{tabular}

The date of RPA are the ratio of peak of each characteristic peak to those of peak 1, respectively. The value is mean $\pm \mathrm{SD}$ ( $\mathrm{n}=11$ for Unprocessed, Charred and Parching Angelica with spice oil, wine and with soil)

particular group although, the absorption intensities of some peaks were different (Fig. 1a, b and Table 3). The correlation coefficients of each chromatogram to their chromatographic, respectively was $0.991 \pm 0.009$ and $0.943 \pm 0.038$ (mean $\pm \mathrm{SD}, \mathrm{n}=11)$. However, the chromatogram of Unprocessed Angelica showed drastic difference from of that of Charred Angelica (Fig. 1a, b and $2 a, b)$. The correlation coefficient of each the eleven Charred Angelica chromatograms to the Unprocessed Angelica simulative mean chromatogram was only (mean $\pm \mathrm{SD}, \mathrm{n}=11$ ) and the correlation coefficient of the simulative mean chromatogram of Unprocessed Angelica to that of Charred Angelica was 0.549. The 3D-projection plot of PCA in three principal components also shows that samples Unprocessed and Charred Angelica are markedly different (Fig. 3). Comparing the chemical components in the Unprocessed and Charred Angelica, compound 9 was the highest peak in the Unprocessed Angelica whilst the peak with retention time at about $3.8 \mathrm{~min}$ was the highest in the Charred Angelica chromatogram. Besides, the RPA of compound 5 in Unprocessed Angelica sample was 7 times higher than that in the Charred Angelica sample and the RPA of compound 8 in Unprocessed Angelica sample was 3 times higher than that in the Charred Angelica sample (Table 3). Thus, Unprocessed and Charred Angelica could be easily distinguished by either chromatograms or the amount of compound 5 and 8 in their samples.

Distinguishing Unprocessed Angelica from parching: Angelica with spice oil, Parching Angelica with wine and Parching Angelica with soil Parching Angelica with spice oil, Parching Angelica with wine and Parching Angelica with soil are related processed products. They are often used as one of the herbal ingredients in the prescriptions of the traditional Chinese medicine. About 11 same components are commonly found in the processed products. Moreover, compound 9 is also the major

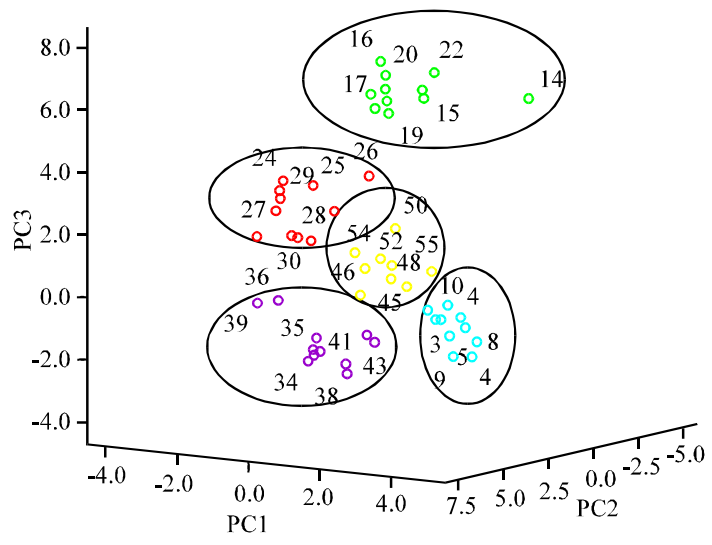

Fig. 3: 3D-projection plots of Principal Component Analysis (PCA) of three principal components for the 55 samples. $\mathrm{PCl}-\mathrm{PC} 3$ are the first three principal components using entire chromatographic profile as input data: 1-11, the samples of Charred Angelica; 12-22, the samples of Parching Angelica with wine; 23-33, the samples of Parching Angelica with soil; 33-44, the samples of Unprocessed Angelica; 44-55, the samples of Parching Angelica with soil

compound in their decoction pieces. Therefore, it was not easy to identify them based on their chemical constituents. Each 11 samples of three processed products were then compared with general consistent chromatograms of each particular processed product (Fig. 1c-e).

The correlation coefficients of each chromatograms of each chromatogram to their simulative mean chromatograms were $(0.995 \pm 0.003)($ mean $\pm \mathrm{SD}, \mathrm{n}=11)$ for Parching Angelica with spice oil, $(0.970 \pm 0.016)$ (mean $\pm \mathrm{SD}$, $\mathrm{n}=11)$ for Parching Angelica with wine and $(0.964 \pm 0.029)$ $(m e a n+S D, n=11)$ for Parching Angelica with soil. However, some of 11 characteristic peaks in their 
chromatograms qualitatively distinguish unprocessed Angelica from Parching Angelica with spice oil, Parching Angelica with wine and Parching Angelica. The RPA of Compound 2, 4-5, 7-8 and 10 in three processed products was markedly lower than that in Unprocessed Angelica but The RPA of Compound 6 and 11 in three processed products were prominent higher than that in Unprocessed Angelica. The correlation coefficient of chromatogram showed that Unprocessed Angelic samples was different from Parching Angelica with spice oil, Parching Angelica with wine and Parching Angelica and the 3D-projection plot of PCA in three principal components also was different between Unprocessed Angelica and three processed products (Fig. 3). However, their difference was nor much significant (Table 2). The correlation coefficient of each Parching Angelica with spice oil to the unprocessed Angelica simulative mean chromatogram $(0.989 \pm 0.008$, mean $\pm \mathrm{SD}, \mathrm{n}=11)$, that of each Parching Angelica with wine to the Unprocessed Angelica simulative mean chromatogram $(0.963 \pm 0.030, \mathrm{n}=11)$ and that of each Parching Angelica with soil to the Unprocessed Angelica simulative mean chromatogram $(0.961 \pm 0.020, \mathrm{n}=11)$ were not significantly smaller than the correlation coefficient of each Unprocessed Angelica chromatogram to the Unprocessed Angelica simulative mean chromatogram $(0.991 \pm 0.009, \mathrm{n}=11)$. This indicated that there was resemblance in terms of chemical constituents of Unprocessed Angelica sample with Parching Angelica with spice oil, Parching Angelica with wine and Parching Angelica sample.

Distinguishing between processed products: Charred Angelica can be easily distinguished from Parching Angelica with spice oil, Parching Angelica with wine and Parching Angelica with soil by either chromatographic patterns or characteristic compounds (Fig. 2b-d and Table 3). The correlation coefficients of Charred Angelica simulative mean chromatogram to Parching Angelica with spice oil, Parching Angelica with wine and Parching Angelica with soil simulative mean chromatogram were only $0.520,0.522$ and 0.561 , respectively (Table 2). Comparing the chemical components in the processed products chromatogram, the peak with retention time at about $3.8 \mathrm{~min}$ was the highest in Charred Angelica chromatogram but compound 9 was the highest peak in the other processed products chromatogram. Meanwhile, the RPA of compounds 2, 4-5 and 8-11 in charred Angelica sample was all less than those in the other processed products samples. Especially, the RPA of compound 5 in Charred Angelica sample was less than about 5-6 times of that of the other processed products samples and the RPA of compound 9 in Charred Angelica sample was less than about 2-3 times of that of the other processed products samples. The chromatograms of Parching Angelica with spice oil, Parching Angelica with wine and Parching Angelica with soil were quite similar (Fig. 1c-e and 2c-e), the correlation coefficient of the simulative mean chromatogram of Parching Angelica with spice oil to Parching Angelica with wine and Parching Angelica was 0.990 and 0.993 , respectively and that of the simulative mean chromatogram of Parching Angelica with wine to Parching Angelica with soil was 0.984 which was close to the correlation coefficient of each Parching Angelica with spice oil chromatogram to their simulative mean chromatogram $(0.995 \pm 0.003$, mean $\pm S D, n=11)$, each Parching Angelica with wine chromatogram to their simulative mean chromatogram $(0.970 \pm 0.016$, mean $\pm \mathrm{SD}$, $\mathrm{n}=11$ ) and Parching Angelica with soil chromatogram to their simulative mean chromatogram (0.964 \pm 0.029 , mean $\pm \mathrm{SD}, \mathrm{n}=11$ ). However, the RPA of compound 9 in Parching Angelica with spice oil samples was significantly higher than that in Parching Angelica with wine and Parching Angelica with soil and the RPA of compound 11 in Parching Angelica with wine was significantly higher than that in Parching Angelica with soil.

Moreover, some difference in fingerprint patterns could be still observed for the processed products samples in the 3D-projection plot of PCA. It is worth noting that the RPA of compound 11 in Parching Angelica with wine was significantly higher than that not only in Parching Angelica with soil but also in Parching Angelica with spice oil, Parching Angelica with soil, Charred Angelica and Unprocessed Angelica. Using this information, it would helpful to distinguishing Parching Angelica with wine from Unprocessed Angelica and the other processed products by comparing the RPA or amount of compound 11 in their chromatograms.

Hierarchical clustering analysis: $\mathrm{HCA}$ is a popular method that can be used to provide a quantitative comparison. Prior to HCA, the RPA of 11 characteristic peaks of all samples were selected for PCA of different processed products. About 4 characteristic principal components obtained from PCA were then used as the input data for $\mathrm{HCA}$ of different processed products. A dendrogram was prepared using Ward Method. The results indicated that 55 samples were divided into five clusters at a rescaled distance of 5 (Fig. 4) and the same processed product samples were clustered in the same cluster. At a rescaled distance of 10, 55 samples formed into 4 groups, Angelica with spice oil and Parching Angelica with soil samples were clustered in the same group. At a rescaled distance of 15, 55 samples were divided into three groups, Parching Angelica with spice oil, Parching Angelica with soil and Charred Angelica samples were combined in the same groups. At a rescaled distance of 20, 55 samples formed into 2 groups, unprocessed products samples were solely divided into one group, different processed products samples were divided into the other group. This indicated that the 


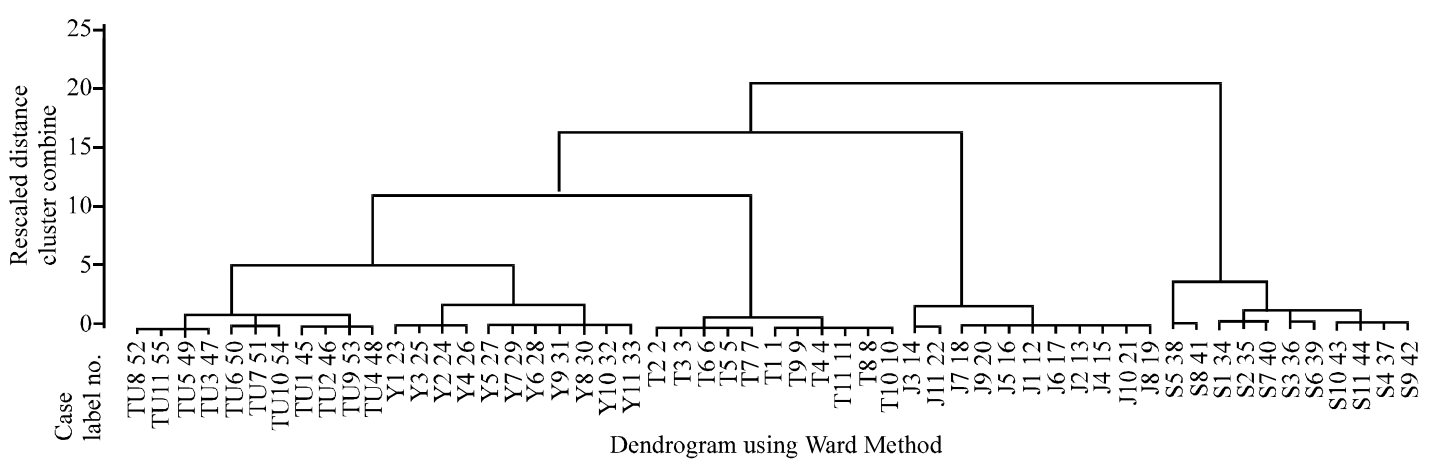

Fig. 4: Hierarchical clustering analysis figure of different processed products

content (The RPA) of 11 characteristic compound of Unprocessed Angelica makes drastic change after processing.

Discriminant analysis: DA can be used to build a predictive model of the group membership based on observed characteristics in each case. This procedure generates a discriminant function (or for $>2$ groups, a set of discriminant functions) based on linear combinations of the predictor variables that provide the best discrimination among the groups. The functions are generated from the samples with known membership; the functions can then be applied to new cases with measurements for the predictor variables but with unknown group membership. The RPA of 11 characteristic peaks was selected from the fingerprints, thereby creating 11 variables. However, not all these variables are of value to the establishment of discriminant function. Only 6 variables, corresponding to the RPA of peaks 2-4, 6, 9 and 11 were selected to generate fisher's discriminant functions using the SPSS software.

$$
\begin{aligned}
\mathrm{T}= & 12.778 \times 2+150.640 \times 3-34.763 \times 4-13.150 \times 6+1.621 \times 9- \\
& 15.933 \times 11-53.66 \\
\mathrm{~J}= & 31.860 \times 2+65.495 \times 3-64.162 \times 4-87.037 \times 6+2.283 \times 9 \\
& +28.043 \times 11-78.794 \\
\mathrm{Y}= & 46.192 \times 2+61.446 \times 3-69.363 \times 4-66.419 \times 6+4.172 \times 9- \\
& 10.877 \times 11-85.214 \\
\mathrm{~S}= & 55.225 \times 2+117.496 \times 3-4.879 \times 4-164.701 \times 6+4.314 \times 9- \\
& 34.865 \times 11-136.785 \\
\mathrm{TU}= & 41.580 \times 2+88.853 \times 3-42.721 \times 4-39.669 \times 6+2.730 \times 9- \\
& 16.419 \times 11-63.190
\end{aligned}
$$

Discriminant standard: Each sample has five functional values and is assigned to the group corresponding to the highest of these function values. T, J, Y, S and TU denote the samples from groups Charred Angelica, Parching Angelica with wine, Parching Angelica with spice oil,

\begin{tabular}{|c|c|c|c|c|c|c|}
\hline \multirow[b]{2}{*}{ Categories } & \multicolumn{5}{|c|}{ Predicted group membership } & \multirow[b]{2}{*}{ Total } \\
\hline & $\mathrm{T}$ & $\mathrm{J}$ & $\mathrm{Y}$ & $\mathrm{S}$ & $\mathrm{TU}$ & \\
\hline \multicolumn{7}{|c|}{ Original (count) } \\
\hline $\mathrm{T}$ & 11 & 0 & 0 & 0 & 0 & 11 \\
\hline $\mathrm{J}$ & 0 & 11 & 0 & 0 & 0 & 11 \\
\hline $\mathrm{Y}$ & 0 & 0 & 11 & 0 & 0 & 11 \\
\hline $\mathrm{S}$ & 0 & 0 & 0 & 11 & 0 & 11 \\
\hline TU & 0 & 0 & 0 & 0 & 11 & 11 \\
\hline \multicolumn{7}{|l|}{ Percentage } \\
\hline $\mathrm{T}$ & 100 & 0 & 0 & 0 & 0 & 100 \\
\hline $\mathrm{J}$ & 0 & 100 & 0 & 0 & 0 & 100 \\
\hline $\mathrm{Y}$ & 0 & 0 & 100 & 0 & 0 & 100 \\
\hline $\mathrm{S}$ & 0 & 0 & 0 & 100 & 0 & 100 \\
\hline$\underline{\mathrm{TU}}$ & 0 & 0 & 0 & 0 & 100 & 100 \\
\hline
\end{tabular}
Unprocessed Angelica and Parching Angelica with soil, respectively and $\mathrm{X}$ denotes the variable. In order to place an unknown sample, the values of the six variables are

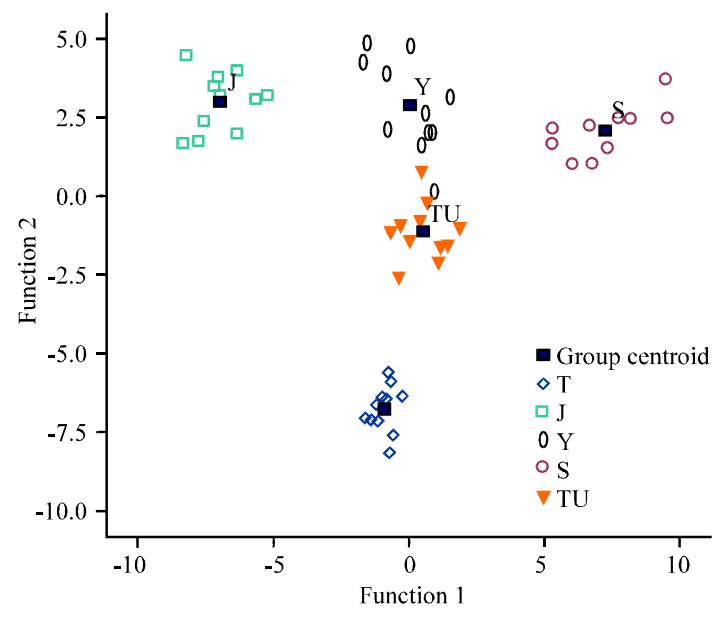

Fig. 5: DA plots for different processed products using canonical discriminant functions S: Unprocessed Angelica; T: Charred Angelica; Y: Parching Angelica with spice oil; J: Parching Angelica with wine and TU: Parching Angelica with soil

inserted into the equations and the sample grouped according to the discriminant standard value obtained. Use of the 6 most discriminating variables enable test samples belonging to groups $\mathrm{T}, \mathrm{J}, \mathrm{Y}, \mathrm{S}$ and $\mathrm{TU}$ to be classified with $100 \%$ accuracy (Table 4 ). The well-resolved DA plots for the 5 groups are shown in Fig. 5. 


\section{CONCLUSION}

HPLC chromatographic fingerprint analysis provides a highly rational approach to the authentication and quality assessment of TCM. Nowadays, chromatographic fingerprints are being widely used in quality control of Angelica and Wang et al. (2006) established a criterion for distinguishing Angelica from the three areas with chromatographic fingerprint combined with discriminant analysis, the accuracy of the discriminant model is as high as $90.0 \%$ which is much better than guessing or traditional methods.

To the knowledge, this is the first report of the effective use of the methodology to distinguish not only different processed products of Angelica but also to differentiate Angelica samples of the same processed product. Furthermore, researchers have applied chemometric techniques to provide a more comprehensive and quantitative analysis of the samples.

The developed HPLC can be used to differentiate and assess different processed products of Angelica. The chemical components in unprocessed Angelica and different processed products were quite different and they be easily distinguished by both their chromatographic patterns and the content of 11 characteristic compounds. Analysis of the 3D-projection plot and $\mathrm{HCA}$ further ensured that the chemical components were quantitatively difference between unprocessed Angelica and different processed products.

DA generated five fish's discriminant functions with the RPA of six selected characteristic peaks for distinguishing different processed products of Angelica, $100 \%$ accuracy and well-resolved DA plots for the 5 groups was obtained from five generated fish's discriminant functions.

Therefore, the developed HPLC chromatographic fingerprint with chemometric techniques (including PCA, $\mathrm{HCA}$ and DA) can provided a more comprehensive and quantitative chemical validation and quality control system. Furthermore, the methodology reported here can also be used to determine relationships between chemical composition and therapeutic effects of Angelica different products.

\section{ACKNOWLEDGEMENT}

Hua Yong-Li and Guo Yan-Sheng are equally contributed to this study.

\section{REFERENCES}

Chen, H.Z., 2008. The research progress of angelica. Strait Pharm. J., 20: 83-85.

Gansu Province Health Department, 1999. Gansu Processing Standard of Traditional Chinese Medicine. 1st Edn., Gansu People Publishers, Lanzhou, Pages: 53.

Lu, G.H., K. Chan, Y.Z. Liang, K. Leung, C.L. Chan, Z.H. Jiang and Z.Z. Zhao, 2005. Development of highperformance liquid chromatographic fingerp rints for distinguishing Chinese Angelica from related umbelliferae herbs. J. Chromatogr. A, 1073: 383 -392.

State Pharmacopoeia Commission of the People's Republic of China, 2005. Pharmacopoeia Commission of the People' s Republic of China. 1st Edn., Chemical Industry Press, Beijing, Pages: 89.

The Compile Commission of Zhonghua Bencao of the People's Republic of China, 1999. Zhonghua Bencao. Vol. 5, Shanghai Science and Technology Press, Shanghai, Pages: 893.

Wang, T.T., X.H. Chen, Q.Q. Hu and K.S. Bi, 2006. RP-HPLC fingerprint for quality assessment of radix angelicae dahuricae. RP-HPLC fingerprint for quality assessment of radix angelicae dahuricae. Acta Pham. Sin., 41: 747-751.

Wu, Y.Y., M.Y. Shang and S.Q. Cai, 2008. HPLC fingerprint of the components of radix angelicae sinensis. Acta Pharm. Sin., 43: 728-732.

Yi, T., H. Zhang, J. Xie and D. Xue, 2007a. A new procedure for the preparative separation and isolation of Z-ligustilide from the roots of angelica sinensis. J. Sep. Sci., 30: 1973-1978.

Yi, T., K.S.Y. Leung, G.H. Lu and H. Zhang, 2007b. Comparative analysis of ligusticum chuangxiong and related umbelliferous medicinal $\mathrm{p}$ lants by high performance liquid chromatography-electrosp ray ionization mass spectrometry. Planta Med., 73: 392-398.

Zhang, M., P. Hu and G.A. Luo, 2007. HPLC fingerprint of the water-soluble constituents in angelica sinensis. Chin. Tradit. PatMed., 29: 628-630. 